\title{
Variability of the Spectrum of the Young Supernova Remnant G11.2-0.3
}

\author{
V. P. Ivanov ${ }^{a} *$, A. V. Ipatov ${ }^{a}$, I. A. Rahimov ${ }^{a}$, and T. S. Andreeva ${ }^{a}$ \\ ${ }^{a}$ Institute of Applied Astronomy, Russian Academy of Sciences, St. Petersburg, 191187 Russia \\ *e-mail: ivanov_vp41@mail.ru
}

Received January 20, 2021; revised March 23, 2021; accepted March 31, 2021

\begin{abstract}
The spectrum of G11.2-0.3 has been refined by bringing the published intensity measurements to the "artificial moon" flux scale, and the dynamics of its changes on different time scales from 0.4 to more than $\sim 50$ years has been investigated. An increase in the fluxes of radio emission of G11.2-0.3 for $\geq 30$ years at $3 \mathrm{~cm} \leq \lambda \leq 375 \mathrm{~cm}$ with a frequency dependence was found: the average rate of changes decreases proportionally to $\log (f)$, and at frequencies $f \geq 10 \mathrm{GHz}$, the increase gave way to a decrease. Measurements with the RT-32 radio telescope of the Svetloe observatory (IAA RAS) in 2013-2019 showed a decrease in fluxes of G11.2-0.3 against the background of rapid nonstationary changes with an average rate of $(-5.4 \pm 6.6) \% /$ year at a wavelength $\lambda=6.2 \mathrm{~cm}$ and $(-1.5 \pm 0.9) \% /$ year at $\lambda=3.5 \mathrm{~cm}$. The stages of growth and decline of fluxes are separated by an epoch $2016.9 \pm 0.6$. The spectrum of G11.2-0.3 is the spectra sum of the shell and the plerion, with each of its parameters determined by the method developed for the 1972.5 epoch. The values of the spectral indices $\alpha 1$ of the shell and $\alpha 2$ of PWN are obtained: $\alpha 1_{1972}=0.77$ and $\alpha 2_{1972}=0.251$. The dynamics of radio emission from the remnant reflects the scenario of interaction between the shock wave and CSM. Possible reasons for evolutionary and non-stationary changes are discussed.
\end{abstract}

Keywords: supernova remnant, plerion, shock wave, radio telescope

DOI: $10.1134 / \mathrm{S} 1063772921080059$

\section{INTRODUCTION}

Supernova remnant (SNR) G11.2-0.3 belongs to type "C" remnants, which are referred as combined, i.e., it has an expanding envelope with a pulsar wind nebula (PWN, plerion) located inside it, surrounding the central pulsar PSR J1811-1925. The plerion and pulsar in G11.2-0.3 were first detected in the X-ray range with the ASCA observations, as reported in $[1,2]$.

G11.2-0.3 is one of the three or four youngest corecollapse supernovae (CC SNR) in the Galaxy. The leftover neutron star from the explosion acts as a pulsar, creating a PWN in the middle of the expanding burst. In [3], a feature of the plerion morphology in the hard X-ray range was noted: the synchrotron radiation source contains two oppositely directed jets that have no analogues in radio emission.

The age of the remnant, according to the measurements of the expansion rate of the nebula in the X-ray range based on the Chandra observations, is estimated within 1400-2400 years [3]. Measurements in the radio-wave range with a high angular resolution revealed the circular symmetry of the outer boundary of G11.2-0.3. The dependence of the flux density $S$ on frequency $f$ is close to a power law in the form
$S(f) \propto f^{-\alpha}$ with the spectral index $\alpha=0.5$ [4]. According to [5], the spectral indices of the shell and plerion are $\alpha_{S}=0.56 \pm 0.02$ and $\alpha_{P}=0.25_{-0.10}^{+0.05}$, respectively, and the flux densities have the following values: $S_{t}=16.6 \pm 0.9 \mathrm{Jy}(\lambda=20 \mathrm{~cm})$ and $8.4 \pm 0.9 \mathrm{Jy}$ $(\lambda=6 \mathrm{~cm}), S_{P}=0.36 \pm 0.23 \mathrm{Jy}(\lambda=20 \mathrm{~cm})$ and $0.32 \pm 0.18$ Jy $(\lambda=6 \mathrm{~cm}), S_{S}=16.2 \pm 1.1$ Jy $(\lambda=$ $20 \mathrm{~cm})$ and $8.0 \pm 1.1 \mathrm{Jy}(\lambda=6 \mathrm{~cm})$, where we introduced the notations: $S_{t}$ is the flux densities of the entire source, $S_{P}$ is the flux densities of plerion, and $S_{S}$ is the flux densities of shell. Based on these data, it is possible to estimate the contribution of the plerion to the total flux of SNR. At wavelengths $\lambda=20$ and $6 \mathrm{~cm}$, it was 2.2 and $3.8 \%$, respectively.

The angular diameter of the shell of G11.2-0.3 is 4' [4], the object is, according to [6], at a distance $d \approx$ $5 \mathrm{kpc}$ from the terrestrial observer; according to other estimates, $d$ can be from $4.4 \mathrm{kpc} \mathrm{[6]} \mathrm{to} 5.5-7 \mathrm{kpc}$ [7].

Based on the SNR morphology according to the VLA observations of G11.2-0.3 with a resolution 3" at two frequencies, the authors of [6] came to the conclusion that G11.2-0.3 is an analogue of Cas A at a later stage of evolution. In [8], published in 1960, I. S. Shklovsky predicted an evolutionary secular flux decrease 
Table 1. Parameters of the RT-32 radio telescope of the "Svetloe" observatory (IAA RAS)

\begin{tabular}{c|c|c|c|c|c|c}
\hline$f, \mathrm{MHz}$ & $T_{\text {rec }}, \mathrm{K}$ & $T_{\text {na }}, \mathrm{K}$ & $T_{\text {sys }}, \mathrm{K}$ & $\begin{array}{c}\Delta f, \\
\mathrm{MHz}\end{array}$ & $\mathrm{SUF}$ & $\begin{array}{c}\mathrm{HPBW}, \\
\operatorname{arcsec}\end{array}$ \\
\hline 1550 & 10 & 38 & 48 & 450 & 0.6 & 21.9 \\
2370 & 10 & 37 & 50 & 450 & 0.48 & 14.6 \\
4840 & 10 & 23 & 33 & 1000 & 0.6 & 6.96 \\
8450 & 12 & 27 & 39 & 1000 & 0.56 & 3.94 \\
\hline
\end{tabular}

We used the notations: $f$ is the frequency; $T_{\text {rec }}$ is the noise temperature of the receiver input; $T_{\text {na }}$ is the noise temperature of the antenna; $T_{\text {sys }}$ is the noise temperature of the system; $\Delta f$ is the receiver bandwidth; SUF is the surface utilization factor; and HPBW is the 0.5 power beamwidth in arc minutes. All feeds are circularly polarized.

in SNRs and showed that in young SNRs, in particular in Cas A, the effect can be measured, which was soon confirmed by observations. It has been established that the radio spectra of young SNRs undergo evolutionary and nonstationary changes [9-13], the study of which is important for understanding the physics of these objects; therefore, the problem of detecting and studying the SNR variability G11.2-0.3 is urgent. The radio observation data of the SNR G11.2-0.3 at different frequencies have been published in several dozen papers. To clarify the spectrum of G11.2-0.3, as well as to identify the dynamics of its evolutionary and nonstationary changes, both further measurements of flux densities and bringing the published data into a single system based on the exact absolute scale of fluxes are required.

The paper presents measurements of flux densities of G11.2-0.3 with the RT-32 radio telescope of the "Svetloe" observatory (IAA RAS), as well as the results of converting published data into a single system based on the "artificial moon" (AM) flux scale [14] for the purpose of refinement of the spectrum of this source and study of its variations.

\section{MEASUREMENTS WITH THE RT-32 RADIO TELESCOPE OF THE "SVETLOE" OBSERVATORY (IAA RAS)}

Measurements of flux densities from SNR G11.20.3 relative to the standards of the flux scale AM were carried out in 2013-2019. The parameters of the fully steerable parabolic radio telescope RT-32 with the diameter of $32 \mathrm{~m}$ at the "Svetloe" observatory (IAA RAS) are given in Table 1 [15-17].

The flux densities of the investigated sources were measured relative to the sources - the standards of the AM flux scale [14]. The AM flux scale is based on absolute measurements by the "artificial moon" method, which is superior in accuracy to other methods and includes more than 15 standard sources with spectra covering the $38 \mathrm{MHz}-200 \mathrm{GHz}$ frequency range. A significant difference from other scales and the advantage of the AM scale is the control of the shape of the source spectra (method of relative spectra), independent of absolute measurements. The AM flux scale is adapted to frequencies up to $200 \mathrm{GHz}$ and more based on the standard spectrum of the Crab nebula, studied in detail by absolute measurements using the "artificial moon" method [10]. The spectrum of the Crab Nebula is power-law. Based on the method of relative spectra, it is shown that the power-law is fulfilled at least up to $200 \mathrm{GHz}$ :

$$
S(f)=S_{0}\left(\frac{f}{f_{0}}\right)^{-\alpha},
$$

where $S(f)$ Jy is the flux density at a frequency $f$ $(\mathrm{MHz}) ; S_{0} \mathrm{Jy}$ is the parameter equal to the flux density at a frequency $f(\mathrm{MHz})$; and $\alpha$ is the spectral index. Average value $\alpha=0.327 \pm 0.002$ and does not depend on time; $\frac{1}{S} \frac{d S}{d t}=(-0.159 \pm 0.024) \% /$ year; $S_{0}=(937 \pm$ 22) Jy at a frequency of $f_{0}=1 \mathrm{GHz}$ for the epoch 1992.7.

The main radio emission standard in the AM flux scale is the extragalactic source 3C295. It is characterized by stable radio emission at wavelengths longer than $1 \mathrm{~cm}$ and small angular dimensions: $5^{\prime \prime} \times 1^{\prime \prime}[18]$. In the scale of AM fluxes, the 3C295 spectrum in the frequency range $1425-8450 \mathrm{MHz}$ is determined by the power-law function (1) with the parameters: $\alpha=1.007$ and $S_{0}=8.249 \mathrm{Jy}$ at a frequency $f_{0}=3500 \mathrm{MHz}$.

The RT-32 radio telescope can measure the ratio of the flux densities of the investigated sources and standards of the AM flux scale at four frequencies: 1550 , 2370, 4840, and $8450 \mathrm{MHz}$. The absolute flux densities of the SNR were obtained from the measured flux ratios of the SNR and the AM scale standards.

The method for correcting data and determining measurement errors is described in detail in [13]. Measurement errors include the root-mean-square deviation of the peak antenna temperature ratios, which did not exceed 3\%, as well as errors of the correction for partial resolution of G11.2-0.3 with the antenna pattern. The method for determining this correction is also described in details in [13]. The correction error for the source resolution depends on the difference between the antenna scan temperature profiles and the approximating Gaussian. In the case of G11.2-0.3, the scan profiles along both axes differ little from Gaussians, and the error in the corrections, which is maximum for the wavelength $\lambda=3.5 \mathrm{~cm}$, did not exceed $2.5 \%$. The profiles were determined by averaging two oppositely directed scans. During the observations, the ON -OFF technique was used. The direction of the source's position angle when pointing at an antenna with circular polarization and circular symmetry of the beam does not require corrections. Correction for atmospheric absorption was introduced in 
the form of a multiplier $e^{\gamma}$, where $\gamma=A_{\lambda} / \sin (h), h$ is the elevation angle (height) of the antenna. For wavelengths $18,13,6.2$, and $3.5 \mathrm{~cm}, A_{\lambda}$ is $0.01,0.011,0.012$, and 0.013 , respectively.

The reason for the error in determining the SNR flux density when compared with the 3C295 standard may be the difference in spectral indices (respectively, $\approx 0.5$ and 1.007 ). In our case, the error does not exceed $0.3 \%$ and no corrections were introduced.

Measurements of flux densities of G11.2-0.3 were performed at frequencies of 4840 and $8450 \mathrm{MHz}$ between April 2013 and October 2019. At both frequencies, measurements were repeated in order to identify changes in the source emission. The flux densities of G11.2-0.3 determined at the frequencies of 4840 and $8450 \mathrm{MHz}$ between the epochs 2013.34 and 2019.89 in the AM flux scale are given in Table 2. Since the measurements were carried out on the same radio telescope and under the same conditions, we have given only random errors in Table 2.

Figure 1 presents the data of Table 2 on a logarithmic scale, which allows us to visually assess the correspondence of time sequences $\log (S)$ to a linear law. $\log (S)$ depends on time linearly if the rate of flux change $\frac{1}{S} \frac{d S}{d t}=$ const.

Figure 2 also illustrates the data of Table 2. However, for a better perception of the details of the observed process, the time interval is limited within 2018.2-2020.0 in Fig. 2.

Linear approximation of the dependence on the wavelength $\lambda=6.2 \mathrm{~cm}$ wave according to the data of Table 2 has the form:

$$
\log _{10}(S)=(48.19 \pm 57.63)-(0.023 \pm 0.029) t .
$$

The same on a wavelength $\lambda=3.5 \mathrm{~cm}$ :

$$
\log _{10}(S)=(13.84 \pm 7.58)-(0.0065 \pm 0.0038) t,
$$

where $t=$ Epoch.

At the wavelength $\lambda=6.2 \mathrm{~cm}$ in the time interval 2017.95-2019.66, the flux density on average decreased at the rate: $d(\ln S(t)) / d t=-(0.054 \pm$ $0.066)$, i.e., $\frac{1}{S} \frac{d S}{d t}=(-5.4 \pm 6.6) \% /$ year, and at the wavelength $\lambda=3.5 \mathrm{~cm}$ in the time interval 2013.342019.89 , the flux density also decreased on average with the rate: $d(\ln S(t)) / d t=-(0.015 \pm 0.009)$, i.e., $\frac{1}{S} \frac{d S}{d t}=(-1.5 \pm 0.9) \% /$ year.

A large-scale in time decrease in flux densities was observed at both frequencies in the indicated time intervals in the presence of significant rapid deviations of the current values $\log (S)$ from the average dependences. The largest deviations $\log (S)$ from the mean values were observed at the wavelength $\lambda=6.2 \mathrm{~cm}$ in
Table 2. Flux densities of SNR G11.2-0.3 according to mea-

\begin{tabular}{|c|c|c|c|}
\hline Epoch & $f, \mathrm{MHz}$ & Sam, Jy & $\sigma, \mathrm{Jy}$ \\
\hline 2013.34 & 8450 & 7.016 & 0.20 \\
\hline 2015.509 & 8450 & 6.543 & 0.19 \\
\hline 2017.949 & 4840 & 8.811 & 0.26 \\
\hline 2017.99 & 4840 & 9.153 & 0.30 \\
\hline 2018.03 & 4840 & 8.703 & 0.25 \\
\hline 2018.209 & 4840 & 8.426 & 0.24 \\
\hline 2018.38 & 8450 & 6.979 & 0.33 \\
\hline 2018.387 & 8450 & 6.846 & 0.20 \\
\hline 2018.39 & 8450 & 6.386 & 0.10 \\
\hline 2018.39 & 4840 & 8.081 & 0.23 \\
\hline 2018.40 & 4840 & 8.895 & 0.28 \\
\hline 2018.518 & 8450 & 6.679 & 0.20 \\
\hline 2018.54 & 8450 & 6.575 & 0.19 \\
\hline 2018.57 & 8450 & 6.758 & 0.21 \\
\hline 2018.74 & 4840 & 11.853 & 0.34 \\
\hline 2018.79 & 8450 & 6.719 & 0.20 \\
\hline 2018.80 & 4840 & 7.945 & 0.32 \\
\hline 2018.81 & 4840 & 8.427 & 0.24 \\
\hline 2018.85 & 8450 & 6.799 & 0.20 \\
\hline 2018.86 & 4840 & 7.977 & 0.27 \\
\hline 2018.89 & 4840 & 7.766 & 0.23 \\
\hline 2018.97 & 4840 & 7.136 & 0.39 \\
\hline 2019.09 & 8450 & 6.611 & 0.59 \\
\hline 2019.11 & 4840 & 7.643 & 0.31 \\
\hline 2019.26 & 8450 & 5.616 & 0.16 \\
\hline 2019.29 & 4840 & 8.309 & 0.30 \\
\hline 2019.37 & 8450 & 6.320 & 0.40 \\
\hline 2019.66 & 4840 & 8.213 & 0.33 \\
\hline 2019.89 & 8450 & 6.236 & 0.18 \\
\hline
\end{tabular}
surements with the RT-32 radio telescope

We used the notations: Epoch is the measurement epoch; $f$ is the frequency; Sam is the flux density (am-artificial moon); and $\sigma$ is the random measurement error.

the epochs $2018.74(+11.7 \sigma)$ and $2018.97(-3.1 \sigma)$, and at the wavelength $\lambda=3.5 \mathrm{~cm}$ in the epoch 2019.26 $(-5.2 \sigma)$.

On the wavelength $\lambda=6.2 \mathrm{~cm}$, in the time interval 2018.74-2019.58, a process of change $S$ relative to the average level was observed from $(+39 \%)$ in the 2018.74 epoch to $(-18 \%)$ in the 2018.97 epoch and reaching the average level in the 2019.58 epoch. A similar, but less detailed picture was observed on the wavelength $\lambda=3.5 \mathrm{~cm}$ : a "dip" in depth $(-16 \%)$ relative to the average level was observed in the 2019.26 epoch. 


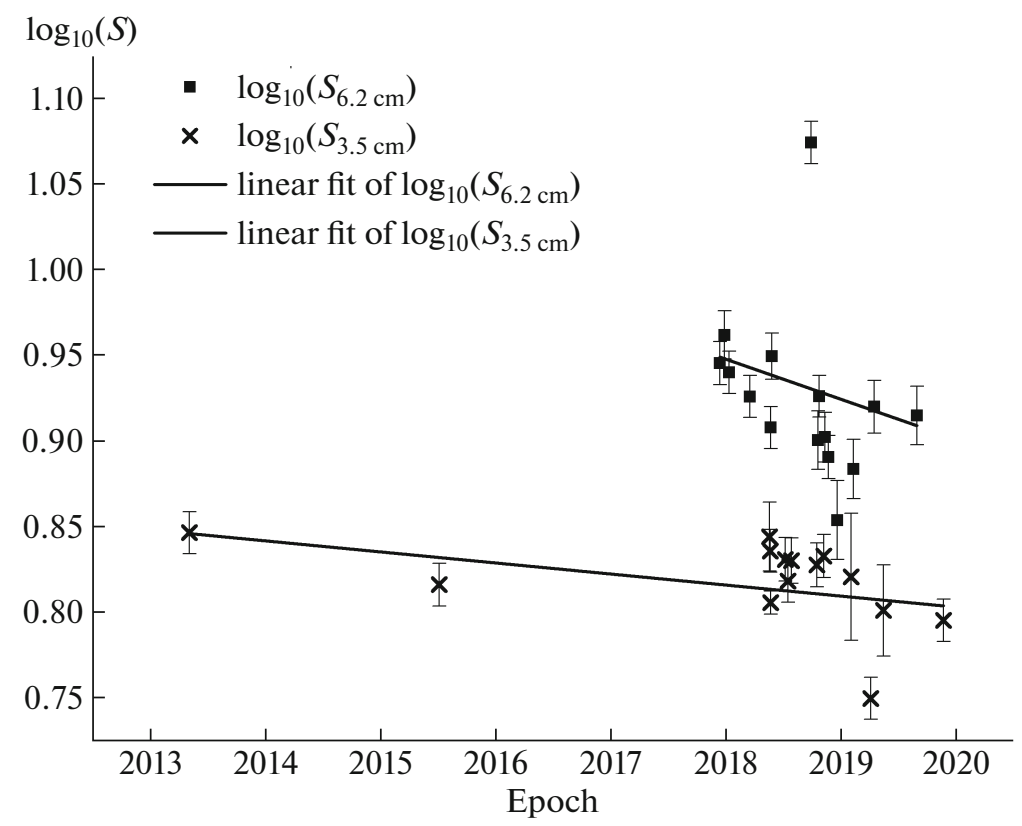

Fig. 1. Logarithms of flux densities of SNR G11.2-0.3 according to Table 2.

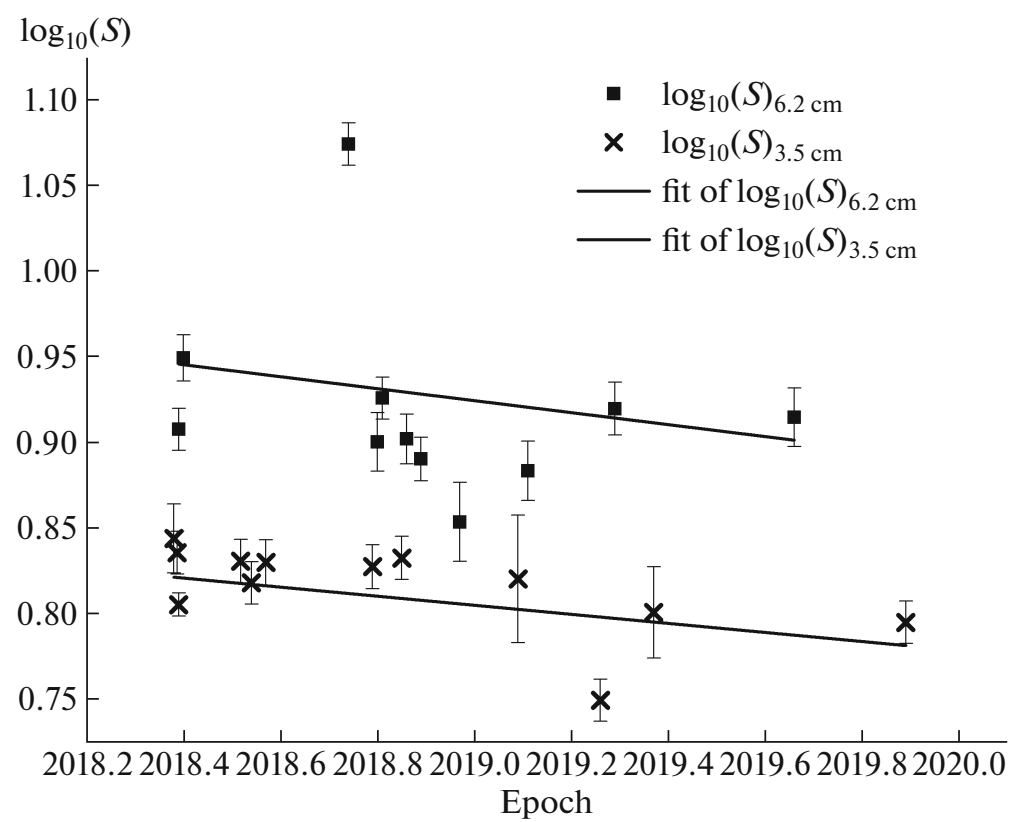

Fig. 2. Distribution of the logarithms of the flux densities of SNR G11.2-0.3 in the time interval 2018.3-2020.0 at wavelengths $\lambda=6.2$ and $3.5 \mathrm{~cm}$ according to the data in Table 2 .

\section{SPECTRUM AND SPECTRAL VARIABILITY OF SNR G11.2-0.3}

In order to refine the spectrum of G11.2-0.3 and study its evolutionary changes over a long-time interval in a wide frequency range, the measurement data published in the literature allowing us to compare the flux densities of G11.2-0.3 and standard sources were reduced by us to the AM flux scale [14]. The advantages of the AM scale over the widely used BGPW scale [19] were discussed in [14]. The flux densities of G11.2-0.3 reduced to the AM flux scale are presented in Table 3. Thus, a unified data system was obtained, defined in the time interval 1966.95-2019.89 in the frequency range $80 \mathrm{MHz}-32 \mathrm{GHz}$. 
Table 3. Flux densities of SNR G11.2-0.3 fluxes according to published data, reduced to the AM flux scale

\begin{tabular}{c|c|c|c|c|c|c|c|c}
\hline$n$ & Epoch & $f, \mathrm{MHz}$ & Sam, Jy & Err, Jy & Sp, Jy & Source & $k$ & References \\
\hline 1 & 1966.95 & 2695 & 9.61 & 0.96 & 11 & $3 \mathrm{C} 274$ & 0.87 & 19 \\
2 & 1967.68 & 5000 & 10.11 & 1.01 & 11 & $3 \mathrm{C} 274$ & 0.92 & 19 \\
3 & 1967.78 & 635 & 12.28 & 1.25 & 13.2 & $3 \mathrm{C} 218$ & 0.93 & 20 \\
4 & 1967.78 & 1410 & 11.89 & 1.15 & 12.6 & $3 \mathrm{C} 218$ & 0.94 & 20 \\
5 & 1967.78 & 2650 & 10.81 & 1.02 & 11.8 & $3 \mathrm{C} 218$ & 0.92 & 20 \\
6 & 1968 & 80 & 36.72 & 4.92 & 36 & Sample & 1.02 & 21 \\
7 & 1968.67 & 5000 & 8.3 & 0.83 & 8.9 & $3 \mathrm{C} 218$ & 0.93 & 22 \\
8 & 1968.67 & 408 & 29.88 & 2.99 & 36 & Sample & 0.83 & 23 \\
9 & 1969 & 2700 & 10.68 & 1.07 & 12 & $19-46$ & 0.89 & 24 \\
10 & 1971 & 80 & 45.33 & 10.3 & 44 & Sample & 1.03 & 25 \\
11 & 1974 & 80 & 54.6 & 16.48 & 53 & Sample & 1.03 & 26 \\
12 & 1974 & 160 & 42.66 & 2.98 & 43 & Sample & 0.99 & 26 \\
13 & 1974.2 & 10630 & 5.1 & 0.7 & 5.8 & $3 \mathrm{C} 123,3 \mathrm{C} 218$ & 0.88 & 27 \\
14 & 1975.5 & 1415 & 13.34 & 0.67 & 14.5 & $3 \mathrm{C} 147$ & 0.92 & 28 \\
15 & 1983.1 & 2695 & 10.18 & 0.44 & 11.49 & $3 \mathrm{C} 286$ & 0.89 & 29 \\
16 & 1984.29 & 1408 & 15.96 & 0.63 & 17.69 & $3 \mathrm{C} 286$ & 0.90 & 30 \\
17 & 1986 & 32000 & 3.39 & 0.2 & 4.04 & $3 \mathrm{C} 286$, NGC 7027 & 0.84 & 31 \\
18 & 1987 & 23000 & 4.34 & 0.46 & 4.7 & NGC 7027 & 0.92 & 32 \\
19 & 1987.87 & 330 & 32.37 & 0.01 & 39 & $3 \mathrm{C} 48,3 \mathrm{C} 286$ & 0.83 & 33 \\
20 & 1996.1 & 14700 & 5.22 & 0.37 & 5.7 & NGC 7027 & 0.92 & 32 \\
21 & 1996.12 & 4850 & 8.59 & 0.45 & 9.6 & $3 \mathrm{C} 286$ & 0.89 & 32 \\
22 & 1999.33 & 32000 & 3.08 & 0.32 & 3.8 & $3 \mathrm{C} 286$ & 0.81 & 32 \\
\hline & 1999.62 & 10450 & 5.79 & 0.37 & 6.3 & $3 \mathrm{C} 286$ & 0.92 & 32 \\
\hline
\end{tabular}

We used the notations: $n$ is the line number; Epoch is the epoch of measurements; $f$ is the measurement frequency; Sam is the flux density and Err is the flux density in the AM flux scale; Sp is the published value of flux density of G11.2-0.3; Source is the source-calibrator (if there are several standard sources-Sample); $k$ are the multipliers of the transition to the AM scale; and we show references to the original literature sources.

Figure 3 shows the flux densities of G11.2-0.3 depending on the frequency according to Table 3.

It should be noted that the measurement errors indicated in the published works contain the uncertainty of the absolute reference, the contribution of which is significant. When these data are presented in the AM flux scale, the errors should decrease due to the elimination of this component, but due to its uncertainty, the errors are preserved. Due to the significant uncertainty of measurement errors, the data in Table 3 were approximated by the power-law dependence of the flux density on frequency in the form (1) without considering the weight of the measurement data. In addition, the point at $635 \mathrm{MHz}$ (1967.78 epoch) with a deviation from the average spectrum by more than $5 \sigma$ was not considered. The approximating power-law function

$$
S_{c}=S_{0}\left(\frac{f}{f_{0}}\right)^{-\alpha}
$$

is obtained with the parameters: $\alpha=0.445 \pm 0.02$; $S_{0}=(16.51 \pm 0.52) \mathrm{Jy}$; and $f_{0}=1 \mathrm{GHz}$.

Figure 4 shows the logarithms of the measured fluxes Sam from Table 3, normalized to calculated values $S_{c}$ at different frequencies depending on time; rationing allows all data to be presented on the same large scale. Changes in flux densities contain a significant non-stationary component, the rates of changes $R=d(\ln S) / d t$ are different at different frequencies. At individual frequencies, they change over time. Esti- 


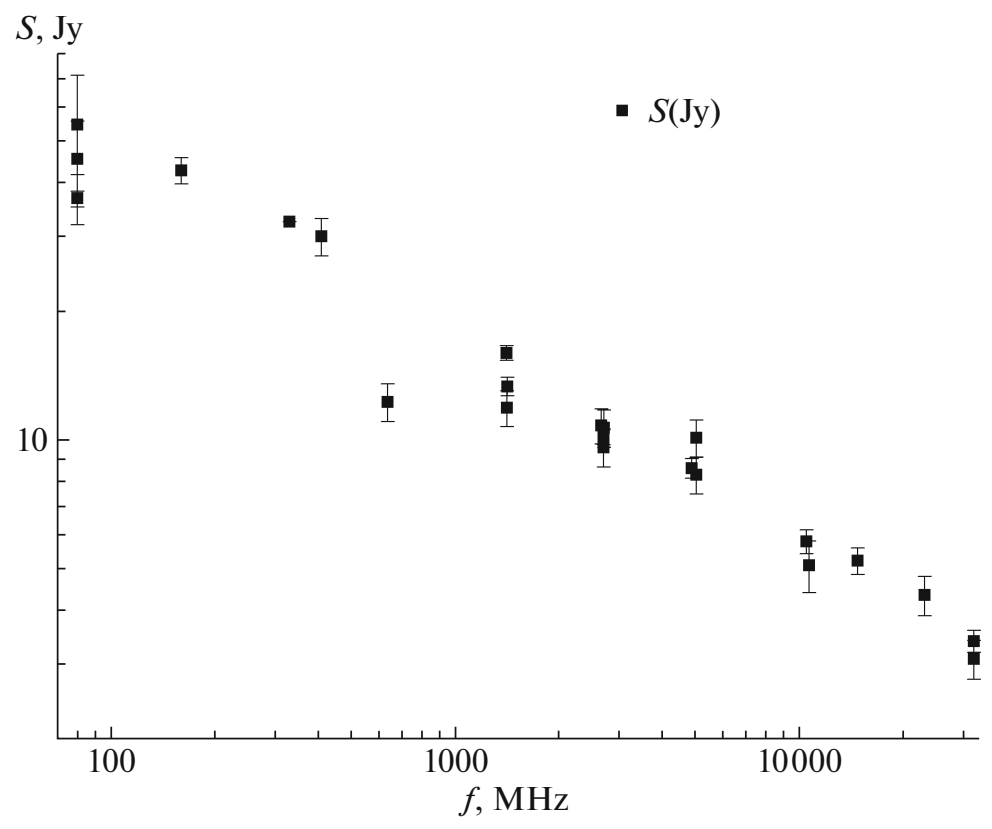

Fig. 3. Flux densities of G11.2-0.3 depending on the frequency according to Table 3.

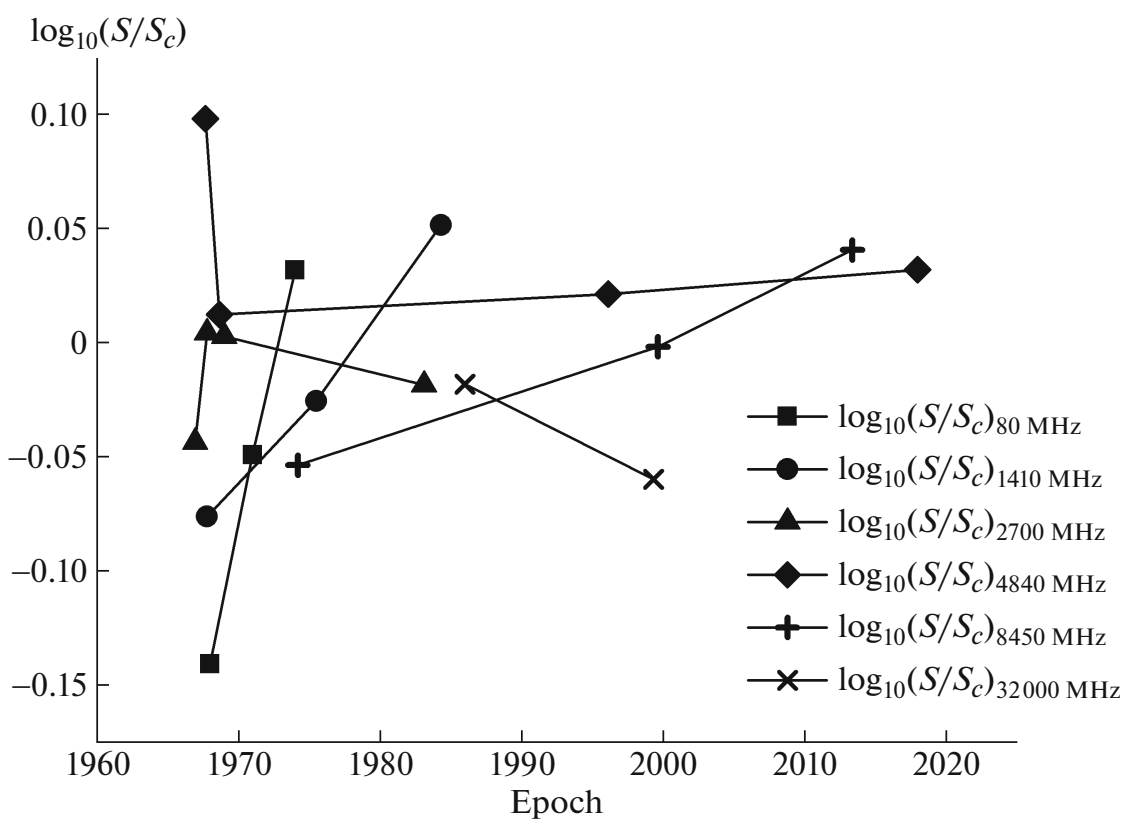

Fig. 4. Logarithms of the ratios of the measured fluxes Sam to the calculated values $S_{c}$ at different frequencies depending on time.

mates of $R$ for most frequencies are limited to time intervals $(6 \leq \Delta t \leq 17)$ years. For most frequencies, they are positive and decrease with increasing frequency. There were "excesses" and "dips" of the flux during short time intervals, which was also noted in the data of our observations with the RT-32.

To determine the parameter $R=d(\ln S) / d t$ at frequencies of $80,1410,4840,8450$, and $32000 \mathrm{MHz}$, the data of Tables 2 and 3 were used, reduced to quoted frequencies based on the value of $\alpha=0.445 \pm 0.002$.

To determine $R(80 \mathrm{MHz}), 80 \mathrm{MHz}$ data of 1968 , 1971 and 1974 epochs were used;

$R(1410 \mathrm{MHz}$ )-data from $1410 \mathrm{MHz}$ (epoch 1967.78), $1415 \mathrm{MHz}$ (epoch 1975.5) and $1408 \mathrm{MHz}$ (epoch 1984.287); 
$R(2695 \mathrm{MHz})-2695 \mathrm{MHz}$ (epoch 1966.95), $2650 \mathrm{MHz}$ (epoch 1967.78), 2.7 MHz (epoch 1969.0), and $2695 \mathrm{MHz}$ (epoch 1983.1) data;

$R(4840 \mathrm{MHz})-5000 \mathrm{MHz}$ (epoch 1967.68), $5000 \mathrm{MHz}$ (epoch 1968.67), $4850 \mathrm{MHz}$ (epoch 1996.12), and $4840 \mathrm{MHz}$ (epoch 2017.95) data;

$R(8450 \mathrm{MHz}$ ) -data $10630 \mathrm{MHz}$ (epoch 1968.67), $10450 \mathrm{MHz}$ (epoch 1999.62) and $8450 \mathrm{MHz}$ (epoch 2013.34); and

$R(32000 \mathrm{MHz})-32000 \mathrm{MHz}$ data in the 1986.0 and 1999.33 epochs.

The parameters of the evolutionary variability of radio emission were determined by comparing not only the flux densities at individual frequencies, but also the current spectra of G11.2-0.3 spaced apart in time. Table 3 shows the measurement data arranged in chronological order and are naturally divided into three groups that combine data that are close in measurement time ( $\leq 8.55$ years). Based on these groups, three current spectra for the mean epochs are determined: 1970.38, 1985.65, and 1997.79. The 1970.38 spectrum includes measurements between 1966.95 and 1975.5 epochs, with the exception of a point at $635 \mathrm{MHz}$ (epoch 1967.78) with a large deviation from the average spectrum, the 1985.65 spectrum is based on measurements from 1983.1 to 1987.87 , and the 1997.79 spectrum includes measurements from 1996.1 to 1999.62 .

The parameters of these spectra were determined in the form (1) without considering the weight of the measurement data; they are given in Table 4.
Table 4. Parameters of the current spectra of G11.2-0.3, calculated according to the data of Table 3

\begin{tabular}{c|c|c|c|c|c|c}
\hline Epoch & $\delta f, \mathrm{GHz}$ & $\alpha$ & $\sigma_{\alpha}$ & $S_{0}, \mathrm{Jy}$ & $\sigma_{S_{0}}, \mathrm{Jy}$ & $\begin{array}{c}f_{0}, \\
\mathrm{GHz}\end{array}$ \\
\hline 1970.38 & $0.08-10.63$ & 0.433 & 0.026 & 16.24 & 0.74 & 1 \\
1985.65 & $0.33-32$ & 0.481 & 0.021 & 18.31 & 0.85 & 1 \\
1997.79 & $4.85-32$ & 0.536 & 0.045 & 20.5 & 2.53 & 1 \\
\hline
\end{tabular}

We show the following: average measurement epoch, Epoch; frequency range, $\delta f$; spectral index, $\alpha$, and its error, $\sigma_{\alpha}$; flux density, $S_{0}$, at frequency $f_{0}$ and its error $\sigma_{S_{0}}$; and frequency $f_{0}$.

Table 4 is illustrated in Figs. 5 and 6, which show the dependences of the spectral index $\alpha$ and parameter $S_{0}$ on time, respectively.

As can be seen from Figs. 5 and 6, the dependences of $\alpha$ and $\log \left(S_{0}\right)$ on time are close to linear:

$$
\begin{gathered}
\alpha(t)=(0.412 \pm 0.009)+(0.00351 \pm 0.0005) t \\
\log \left(S_{0}(t)\right)=(1.191 \pm 0.003) \\
+(0.00354 \pm 0.0002) t
\end{gathered}
$$

where $t=($ Epoch -1965.0$)$.

From (1), it follows:

$$
\begin{gathered}
\log (S)=\log \left(S_{0}\right)-\alpha \log \left(f / f_{0}\right), \\
\ln (S(f, t))=2.3[(1.191+0.00354 t) \\
\left.-(0.412+0.00351 t) \log \left(f / f_{0}\right)\right] .
\end{gathered}
$$

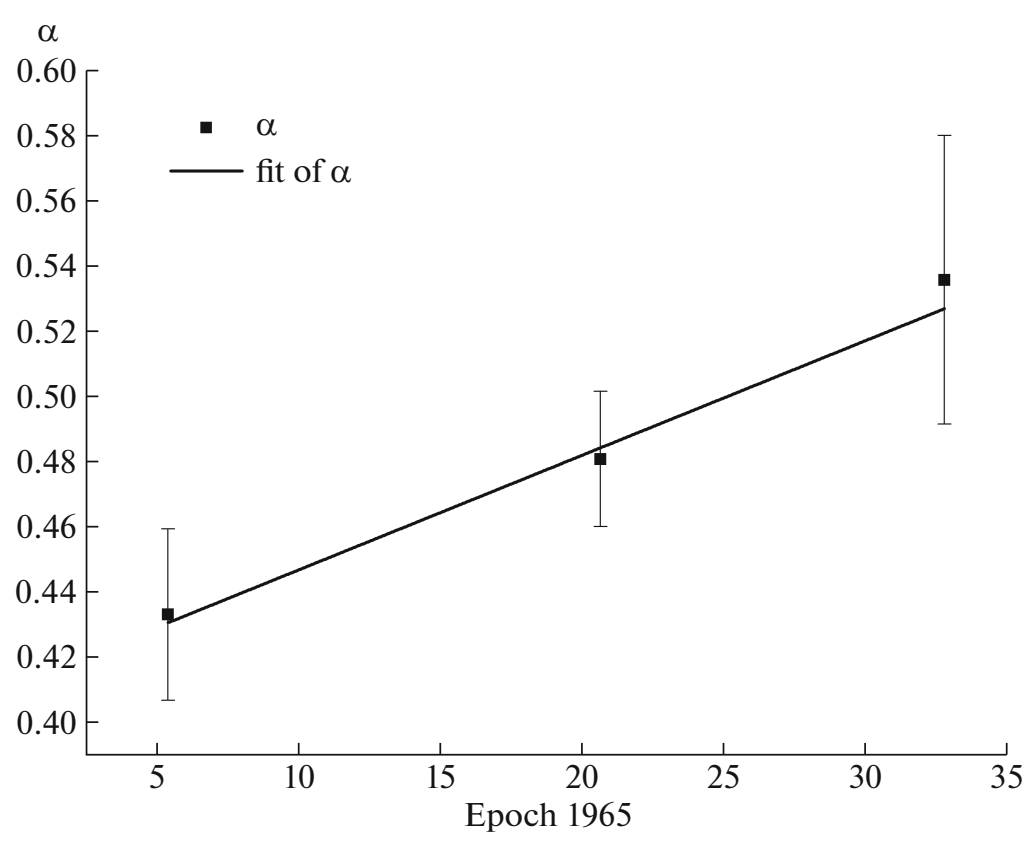

Fig. 5. Dependence of the spectral index $\alpha$ on time. 


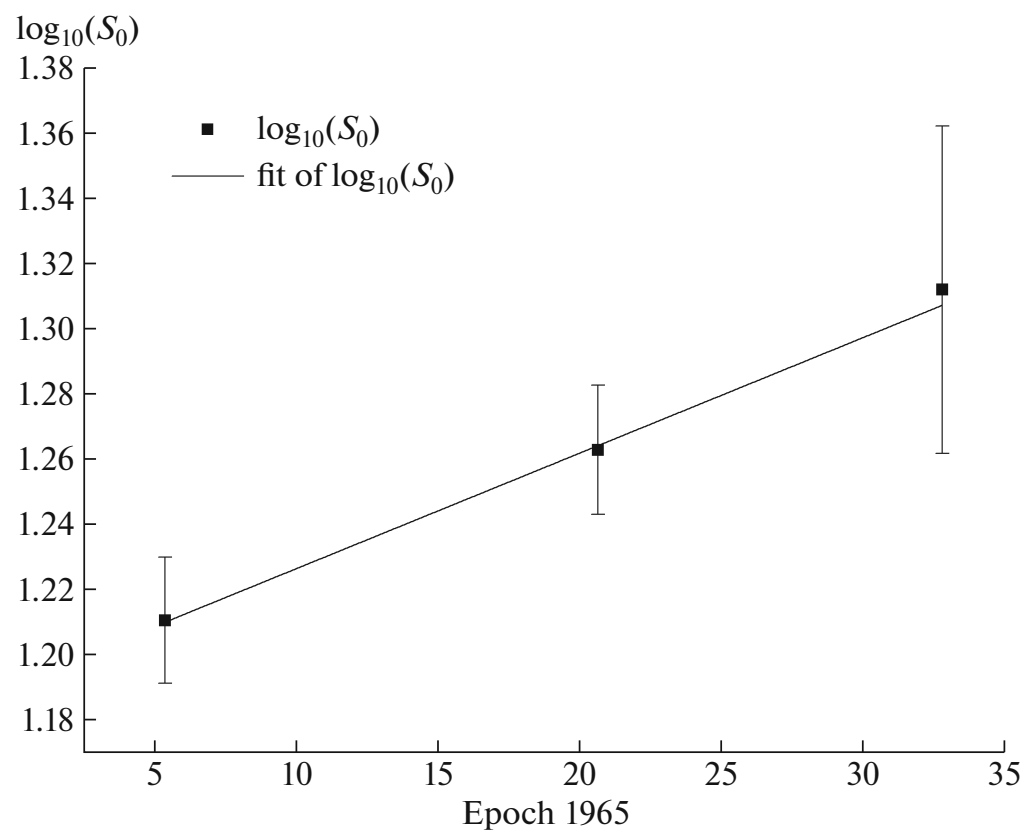

Fig. 6. Dependence of the parameter $S_{0}$ on time.

The rate of flux change $R=\frac{1}{S} \frac{d S}{d t}=d(\ln S) / d t$. Differentiating (4), we determine

$$
R=\frac{1}{S} \frac{d S}{d t}=2.3\left(0.00354-0.00351 \log \left(f / f_{0}\right)\right)
$$

Average rate of flux change in the time interval 1967-2000 depends on frequency: an increase in the flux was observed at frequencies below $10.2 \mathrm{GHz}$; at higher frequencies, the flux decreased. The values of frequencies $f$ [MHz], velocities $R$ [\%/year], errors of determination for $R, \sigma R$ [\%/year], and the time interval $\Delta t$ are given in Table 5 .

The spectral dependence is determined by relation (8), or its equivalent:

$$
R[\% / \text { year }]=3.236-0.807 \log (f[\mathrm{MHz}]) .
$$

Figure 7 shows the rates of change in the fluxes of SNR G11.2-0.3, determined from the current spectra and from measurements at one frequency according to the data in Table 5.

In Fig. 7, we see that the rates of flux change, determined at individual frequencies and by spectra, are different within the error limits, with the exception of the frequency $80 \mathrm{MHz}$, where the intervals $\Delta t$ in which the values $R$ were determined are the most different.

Using the relation ( $\left.8^{\prime}\right)$, the data in Table 3 can be adjusted to a common epoch to compensate for largescale variability. For the epoch 1972.5, a spectrum was obtained with the parameters:

$$
\begin{gathered}
\alpha_{1972}=0.497 \pm 0.02, \\
\left(S_{0}\right)_{1972}=(18.731 \pm 0.878) \mathrm{Jy} .
\end{gathered}
$$

Table 5. Rate of flux change of SNR G11.2-0.3, determined from measurements at one frequency and from the current spectra

\begin{tabular}{r|c|c|c|c|c|c}
\hline \multirow{2}{*}{$f, \mathrm{MHz}$} & \multicolumn{3}{|c|}{ At one frequency } & \multicolumn{3}{c}{ From spectra } \\
\cline { 2 - 7 } & $R, \% /$ year & $\sigma R, \% /$ year & $\Delta t$ & $R, \% /$ year & $\sigma R, \% /$ year & $\Delta t$ \\
\hline 80 & 6.70 & 0.02 & $1968.0-1974.0$ & 1.7 & 0.82 & $1966.95-1999.62$ \\
1410 & 1.89 & -0.01 & $1967.78-1984.29$ & 0.69 & 0.81 & $1966.95-1999.62$ \\
2695 & -0.1 & & $1966.95-1983.1$ & 0.47 & 0.82 & $1966.95-1999.62$ \\
4840 & -0.06 & -0.01 & $1967.68-2017.95$ & 0.26 & 0.82 & $1966.95-1999.62$ \\
8450 & 0.97 & 0.01 & $1974.2-2013.34$ & 0.07 & 0.82 & $1966.95-1999.62$ \\
32000 & -0.72 & & $1986.0-1999.33$ & -0.4 & 0.83 & $1966.95-1999.62$ \\
\hline
\end{tabular}

We show the following: frequency, $f$; results obtained at one frequency-the average rate of flux change $R$ in the time interval $\Delta t$ and its error $\sigma R$ in the same interval, the used time interval $\Delta t$ (start-end); the results obtained from the spectra-the rate of flux change $R$ and the error of its determination $\sigma R$, the time interval $\Delta t$ at which the parameter $R$ was determined. 


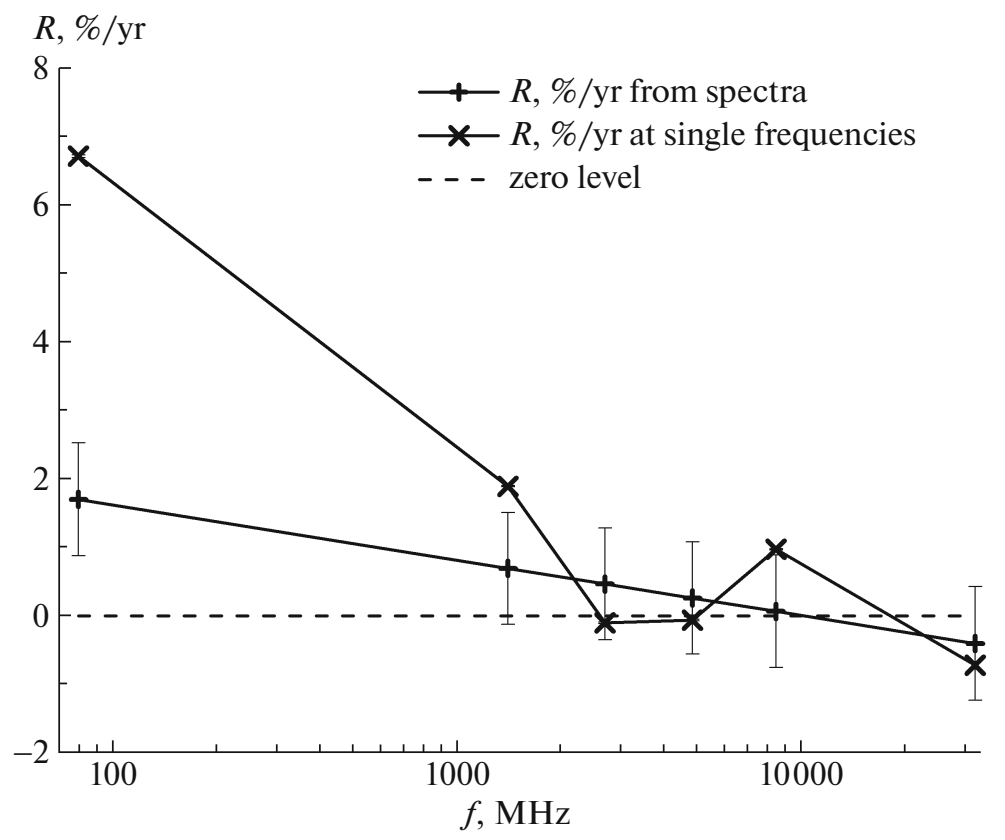

Fig. 7. Rates of flux change of SNR G11.2-0.3, determined from the current spectra and measurements at the single frequencies.

The combined SNR G11.2-0.3 consists of a shell and a plerion with a pulsar in the center. The spectra of the shell and plerion are power-law spectra with different spectral indices, which make it possible to divide the total spectrum of this source with a sufficiently accurate determination into two parts corresponding to the components of the source. The technique is described in [13], where it was applied to SNR 3C396. In short, the process is as follows.

1. To refine the spectrum of the combined source, the logarithms of the fluxes measured at close frequencies were averaged; the mean logarithm of the flux corresponds to the mean logarithm of the frequency. The averaging did not consider data with rapid deviations from the mean of more than $2 \sigma$. The averaging process minimizes the effect of rapid variability and measurement errors. The refined spectrum does not include once-measured data or data with signatures of rapid changes over time. Table 6 shows the spectrum for the epoch 1972.5. The function $\langle\log (\operatorname{Sam})\rangle=F(\log (f))$ is approximated by a polynomial of the second degree.

Table 6 represents data averaged over the following frequency ranges

$i=1$, the frequencies 1410,1415 , and $1408 \mathrm{MHz}$

$i=2$, the frequencies 2695, 2650, 2700, and $2695 \mathrm{MHz}$;

$i=3$, the frequencies 5000 and $4850 \mathrm{MHz}$;

$i=4$, the frequencies 10630 and $10450 \mathrm{MHz}$; and

$i=5$, the frequencies 32000 and $32000 \mathrm{MHz}$.
2. We denote by $\mathrm{S}_{1_{c}}$ and $\mathrm{S}_{2_{c}}$ the spectra of components 1 and 2 (a shell and a plerion, respectively), and by $\left\langle\operatorname{Sam}_{i}\right\rangle$ the averaged flux densities at frequencies $f_{i}$. The parameters of one of the two components of the spectrum, $\alpha 1$ and $S_{01}$, are set arbitrarily, and the calculation of flux densities $S_{1_{c}}\left(f_{i}\right)$ at frequencies $f_{i}$ is performed according to the formula:

$$
S_{1_{c}}\left(f_{i}\right)=S_{01}\left(\frac{f_{i}}{f_{0}}\right)^{-\alpha 1} .
$$

The optimal parameters at which the root-meansquare deviation of the sum of the calculated values

Table 6. Average values $\langle$ Sam $\rangle$ of measured flux densities reduced to epoch 1972.5

\begin{tabular}{c|c|c|c|c}
\hline$i$ & $\left\langle f_{i}\right\rangle, \mathrm{MHz}$ & $\begin{array}{c}\left\langle\mathrm{Sam}_{1972, i}\right\rangle, \\
\mathrm{Jy}\end{array}$ & Err, Jy & $n$ (Table 3) \\
\hline 1 & 1411 & 16.12 & 0.6 & $4,14,16$ \\
2 & 2685 & 11.77 & 0.52 & $1,5,9,15$ \\
3 & 4924 & 8.22 & 0.67 & 7,21 \\
4 & 10540 & 5.44 & 0.56 & 13,23 \\
5 & 32000 & 3.51 & 0.27 & 17,22 \\
\hline
\end{tabular}

We show the following: the number $i$ of the averaging interval for flux densities and frequencies (see text); average frequency of measurements in the $i$-th interval $\left\langle f_{i}\right\rangle$; the average value of the flux density in the $i$-th frequency interval $\left\langle\operatorname{Sam}_{1972, i}\right\rangle$ and its error Err; we listed the line numbers of Table 3, included in the $i$ th interval and containing information about the averaged data. 
$S_{1_{c}}+S_{2_{c}}$ from the values $\log \left\langle\operatorname{Sam}_{1972, i}\right\rangle$ reaches a minimum were determined according to the following scheme. The flux densities of the second component $S_{2_{c}}$ at frequencies $f_{i}$ are determined as the difference $\left\langle\operatorname{Sam}_{i}\right\rangle-S_{1_{c}}\left(f_{i}\right)$, and the parameters of the power-law dependence $S_{2_{c}}(f), \alpha 2$, and $S_{02}$ are determined as a fit of the power-law function for sampling the values of the differences:

$$
S_{2_{c}}\left(f_{i}\right)=S_{02}\left(\frac{f_{i}}{f_{0}}\right)^{-\alpha 2} .
$$

The root-mean-square deviation $\sigma$ of the two-component model is determined by a set of comparisons of the quadratic approximation $\left\langle\mathrm{Sam}_{i}\right\rangle$ from Table 6 and the sums $S_{\Sigma}\left(f_{i}\right)=S_{1_{c}}\left(f_{i}\right)+S_{2_{c}}\left(f_{i}\right)$ calculated according to (9) and (10). The pair of values $\alpha 1$ and $S_{01}$ uniquely correspond to $\alpha 2$ and $S_{02}$, with their change the root-mean-square error of the two-component model changes, reaching a minimum at optimal values of the parameters $\alpha 1, S_{01}, \alpha 2$, and $S_{02}$.

For the epoch 1972.5, the following parameter values were obtained:

$$
\begin{gathered}
\alpha 1=0.77 \pm 0.027 \\
S_{01}=14.4 \pm 0.5 \mathrm{Jy} ; \\
\alpha 2=0.251 \pm 0.029 \\
S_{02}=5.9 \pm 1.1 \mathrm{Jy} .
\end{gathered}
$$

\section{DISCUSSION}

We obtained a unified data system on the frequency-time distribution of the source radio emission intensity in the time interval 1966.95-1999.62 at frequencies from $80 \mathrm{MHz}$ to $32 \mathrm{GHz}$ using the "artificial moon" (AM) flux scale published data of measurements of flux densities SNR G11.2-0.3. Radiation variability was found on time scales from 0.8 to $\sim 50$ years. Evolutionary (averaged) flux changes occur against the background of their rapid nonstationary variability (Fig. 4) and are described by expression (7). The flux density at the frequency $f$ changed with an average rate decreasing proportionally to $\log \left(f / f_{0}\right)$, where $f_{0}=1000 \mathrm{MHz}$. At frequencies $(80-10000) \mathrm{MHz}$, the flux densities increased, while at higher frequencies they decreased with time.

The intensities of SNR G11.2-0.3 were regularly measured at wavelengths $\lambda=6.2$ and $3.5 \mathrm{~cm}$ during 2013-2019 with the RT-32 radio telescope of the "Svetloe" observatory (IAA RAS). Decreasing time sequences of flux densities were obtained (Figs. 1 and 2). At both wavelengths, significant non-stationary deviations of the data from the linear fit were observed. Average rates of flux decrease were $(-5.4 \pm$ $6.6)$ and $(-1.5 \pm 0.9) \% /$ year at waves $\lambda=6.2$ and $3.5 \mathrm{~cm}$, respectively. Time sequences are approxi- mated by linear relations (2) and (3), which in the history of the evolution of the source are a continuation in time of the previously occurring processes described by relation (7). Time dependences (7) go into (2) and (3) in the epochs 2017.5 for $\lambda=6.2 \mathrm{~cm}$ and 2016.3 for $\lambda=3.5 \mathrm{~cm}$, where the growth of fluxes was replaced by their fall.

I.S. Shklovsky showed that in young SNRs expanding in a homogeneous interstellar medium, the intensity of radio emission over time experiences an evolutionary decrease, which he called secular decrease [8]. Observations of historical SNRs have confirmed the predicted effect (e.g., $[9,10])$. The evolution of G11.2-0.3 radiation follows a different scenario.

Although the increase in fluxes in G11.2-0.3 cannot be classified as typical, it is no exception: an increase in the radio emission power is observed in the very young SNRs G1.9+0.3 and 1987A in the Large Magellanic Cloud [34, 35]. The sources G11.2-0.3 and 1987A belong to the core-collapse SNRs and have many similar features, but it is important to note the following: in both cases, the shock wave of the explosion propagates in a strongly inhomogeneous circumstellar medium (CSM) with a density increasing with distance from the center. The radio emission of SNR 1987A grows exponentially, indicating that the propagating shock wave and the associated increasing shock volume interact with the increasingly dense CSM region. Synchrotron radiation is associated with the acceleration of particles as a result of the first-order Fermi mechanism, a process in which particles move between the upstream and downstream regions of a direct shock wave, gaining energy [35].

Based on X-ray morphology, the authors of [3] classify G11.2-0.3 as a SNR formed as a result of an explosion after the loss of most of the progenitor's hydrogen shell (several solar masses) before the explosion. After the explosion, the supernova shock wave collided with this lost mass, both radially and azimuthally inhomogeneous, and the growth of the SNR shell flux is due to the acceleration of electrons at the front of the direct shock wave with increasing efficiency.

The dynamics of the SNR 1987A and G11.2-0.3 spectra is different: the spectrum of G11.2-0.3 became steeper with time (Table 4), in contrast to the 1987A and Cas A spectra, which become flatter with time. In the last two cases, the direct shock wave and the environment interact, in contrast to G11.2-0.3, where it is necessary to consider the interaction of the plerion and the backward shock wave, which, according to [3], reached the center of the remnant. It can be expected that the slope of the spectrum of G11.2-0.3 shell decreases with time, similar to SNR 1987A, and the overall increase in the spectral index is due to the contribution of the plerion exposed to the backward shock wave. As a result of compression by the backward 
shock wave, the magnetic field in the central volume of the remnant should increase. In this case, synchrotron radiation also increases, but at the same time, energy losses for synchrotron radiation of relativistic particles increase [36]. This effect can be observed as a break in the spectrum. According to [37], extrapolation of the PWN power spectrum in the X-ray range on the radio-wave band leads to a "break" frequency of $\sim 8 \mathrm{GHz}$ with an increase in the spectral index by 0.5 , the expected value from synchrotron losses in a homogeneous source with constant electron injection. To implement such a scenario, with an assumed age of the remnant of 2000 years, a magnetic field strength of about $3 \mathrm{mG}$ is required. If the break in the spectrum at a frequency $f_{b} \sim 8 \mathrm{GHz}$ is due to synchrotron losses, then the flux of radio emission from the plerion at frequencies $f \geq f_{b}$ should decrease with time, and the average spectral index in the frequency range 0.08$32 \mathrm{GHz}$ should increase.

The listed observational facts and related conclusions can be compared with our results of determining the parameters of the shell and PWN spectra.

At a frequency of $1410 \mathrm{MHz}$, the plerion contribution to the total flux of G11.2-0.3 in 1972 was $34 \%$. This estimate can be compared with the estimates obtained in [5] given in Section 1. The estimates [5] refer to the 1985.0 epoch. Our spectral index of the plerion $\alpha 2_{1972}$ coincides with $\alpha_{P}$ [5], although the difference between our $\alpha 1_{1972}$ and $\alpha_{S}$ of the shell [5] goes beyond the error limits. However, estimates of the PWN contribution to the total residual flux differ markedly. At a wavelength $\lambda=20 \mathrm{~cm}$ in the epoch 1972.5, we estimate the ratio $S_{P} / S_{t}=0.34$ in contrast to $S_{P} / S_{t}=0.02$ according to [5]. To find out which result is more accurate, it is necessary to identify the component of the source in which fast and deep "dips" of radio emission can occur. The duration of the process imposes a limitation on the linear size of the region in which it operates. During observations with the RT-32 radio telescope (Section 2), dips relative to the mean values at wavelengths $\lambda=3.5$ and $6.2 \mathrm{~cm}$ were noted in 16 and $18 \%$, respectively, in the intervals $\Delta t \leq 0.4$ years. Table 3 contains data on "dips" at frequencies $f=80 \mathrm{MHz}(-17 \%)$ and $f=$ $635 \mathrm{MHz}(-28 \%)$ relative to the average spectrum, which lasted no more than $4-5$ years. At a distance of $5 \mathrm{kpc}$, the shell of G11.2-0.3 has a diameter of $5.8 \mathrm{pc}$ (19 light years), which limits the minimum characteristic time of nonstationary variability of its radiation and excludes the localization of all observed "dips" in the shell. Consequently, the "dips" are associated with the PWN radiation and cannot exceed the contribution of this component to the total SNR flux. Our estimates are consistent with this condition, in contrast to [5].

Rapid nonstationary flux changes noted during observations of G11.2-0.3 with the RT-32 radio tele- scope of the "Svetloe" observatory (IAA RAS) have features common to many plerions. They were previously noted in 3C58 [11], G21.5-0.9 [12], and 3C396 [13]. These sources contain fluxes of relativistic particles and magnetic fields injected by the pulsar. The dynamics of the processes has an analogy with solar flares, the physical mechanism of which is based on reconnection of the magnetic field lines with a rapid release of the magnetic field energy and a burst of radio emission power, followed by its short-term weakening. Rapid nonstationary changes in the radio emission of plerions can be caused by reconnection of the magnetic field lines.

An alternative explanation for the rapid nonstationary changes in radio emission was proposed in [13]. It is assumed that the radio emission from compact sources located in the PWN can interact with the shell acting as a random phase screen and creating a diffraction pattern in the plane of the observer. The variability of this pattern is due to its own motions in the shell.

Another possible reason for deviations from the power law in the radio emission spectra of plerions can be similar deviations in the energy spectra of emitting relativistic electrons injected by the pulsar into the nebula.

\section{CONCLUSIONS}

In the present work, we investigated the spectrum and variability of the radio emission of SNR G11.20.3 . The use of the "artificial moon" flux scale as a basis for combining the measurement data of flux densities of G11.2-0.3 accumulated over more than 50 years of observations, as well as our own observations, made it possible to clarify the source spectrum and the dynamics of its changes in time. As a result:

- unusual properties of the evolution of G11.2-0.3 have been found-an increase in fluxes in a wide frequency band over a long time interval. It is unusual that earlier the growth of fluxes was observed only in very young remnants-G1.9+0.3 and 1987A, while G11.2-0.3 is in an advanced stage of evolution-close to the Sedov phase at an age of $\sim 2000$ years;

- the growth of fluxes at wavelengths $\lambda=6.2$ and $3.5 \mathrm{~cm}$ gave way to a fall in the epoch $2016.9 \pm 0.6$. Changes in the dynamics of radio emission from the remnant can be caused either by the emergence of the leading edge of the shock wave outside the region with a high CSM density, or by the weakening of the effect of the backward shock wave on the PWN; and

- the parameters of the spectra of the components of the composite SNR-shell and PWN were determined, as well as their changes in time were investigated.

Data on fast non-stationary changes in the intensity of radio emission of G11.2-0.3 were obtained. Possible physical mechanisms of the phenomenon can 
be: reconnection of magnetic field lines, diffraction of compact sources located in the PWN on a random phase screen, which is the SNR shell, as well as deviations from the power law in the energy spectra of emitting relativistic electrons injected by the pulsar into the nebula. However, this issue needs further research.

According to the unique properties of SNR G11.20.3 , it can be assumed that further observations and theoretical studies of this object will be effective.

\section{OPEN ACCESS}

This article is licensed under a Creative Commons Attribution 4.0 International License, which permits use, sharing, adaptation, distribution and reproduction in any medium or format, as long as you give appropriate credit to the original author(s) and the source, provide a link to the Creative Commons licence, and indicate if changes were made. The images or other third party material in this article are included in the article's Creative Commons licence, unless indicated otherwise in a credit line to the material. If material is not included in the article's Creative Commons licence and your intended use is not permitted by statutory regulation or exceeds the permitted use, you will need to obtain permission directly from the copyright holder. To view a copy of this licence, visit http://creativecommons.org/licenses/by/4.0/.

\section{REFERENCES}

1. G. Vasisht, T. Aoki, T. Detani, S. R. Kulkarni, and F. Nagase, Astrophys. J. 456, L59 (1996).

2. K. Torii, H. Tsunemi, T. Dotani, and K. Mitsuda, Astrophys. J. 489, L145 (1997).

3. K. J. Borkowski, S. P. Reynolds, and M. S. E. Roberts, Astrophys. J. 819, 160 (2016).

4. D. A. Green, Bull. Astron. Soc. India 42, 47 (2014).

5. C. Tam, M. S. E. Roberts, and V. M. Kaspi, Astrophys. J. 572, 202 (2002).

6. D. A. Green, S. F. Gull, S. M. Tan, and A. J. B. Simon, Mon. Not. R. Astron. Soc. 231, 735 (1988).

7. A. H. Minter, F. Camilo, S. M. Ransom, J. P. Halpern, and N. Zimmerman, Astrophys. J. 676, 1189 (2008).

8. I. S. Shklovskii, Sov. Astron. 4, 243 (1960).

9. K. S. Stankevich, V. P. Ivanov, and S. P. Stolyarov, Astron. Lett. 25, 501 (1999).

10. V. P. Ivanov, K. S. Stankevich, and S. P. Stolyarov, Astron. Rep. 38, 654 (1994).

11. V. P. Ivanov, A. V. Ipatov, I. A. Rakhimov, and T. S. Andreeva, Astrophys. Bull. 74, 128 (2019).

12. V. P. Ivanov, A. V. Ipatov, I. A. Rakhimov, S. A. Grenkov, and T. S. Andreeva, Astron. Rep. 63, 642 (2019).

13. V. P. Ivanov, A. V. Ipatov, I. A. Rakhimov, and T. S. Andreeva, Astron. Rep. 64, 651 (2020).

14. V. P. Ivanov, A. V. Ipatov, I. A. Rakhimov, S. A. Grenkov, and T. S. Andreeva, Astron. Rep. 62, 574 (2018).
15. A. M. Finkel'shtein, Nauka Ross. 5, 20 (2001).

16. A. Finkelstein, A. Ipatov, and S. Smolentsev, in Proceedings of the 4th APSGP Workshop, Ed. by $\mathrm{H}$. Cheng and Q. Zhihan (Shanghai Sci. Tech. Publ., Shanghai, 2002), p. 47.

17. I. A. Rakhimov, Sh. B. Akhmedov, A. A. Zborovskii, D. V. Ivanov, A. V. Ipatov, S. G. Smolentsev, and A. M. Finkel'shtein, in Proceedings of the All-Russia Astronomical Conference (IPA RAN, St. Petersburg, 2001), p. 152.

18. M. Ott, A. Witzel, A. Quirrenbach, T. P. Krichbaum, K. J. Standke, C. J. Schalinski, and C. A. Hummel, Astron. Astrophys. 284, 331 (1994).

19. W. J. Altenhoff, D. Downes, L. Goad, A. Maxwell, and R. Rinehart, AG11.2-0.3stron. Astrophys. Suppl. Ser. 1, 319 (1970).

20. D. K. Milne, T. L. Wilson, F. F. Gardner, and P. G. Mezger, Astrophys. Lett. 4, 121 (1969).

21. O. B. Slee and C. S. Higgins, Austral. J. Phys. Astrophys. Suppl. 27, 1 (1973).

22. W. M. Goss and P. A. Shaver, Austral. J. Phys. Astrophys. Suppl. 14, 1 (1970).

23. P. A. Shaver, W. M. Goss, Austral. J. Phys. Astrophys. Suppl. 14, 77 (1970).

24. W. M. Goss and G. A. Day, Austral. J. Phys. Astrophys. Suppl. 13, 3 (1970).

25. G. A. Dulk and O. B. Slee, Austral. J. Phys. 25, 429 (1972).

26. G. A. Dulk and O. B. Slee, Astrophys. J. 199, 61 (1975).

27. R. H. Becker and M. R. Kundu, Astron. J. 80, 679 (1975).

28. P. A. Shaver and K. W. Weiler, Astron. Astrophys. 53, 237 (1976).

29. W. Reich, E. Furst, P. Steffen, K. Reif, and C. G. T. Haslam, Astron. Astrophys. Suppl. Ser. 58, 197 (1984).

30. W. Reich, R. Reich, and E. Furst, Astron. Astrophys. Suppl. Ser. 83, 539 (1990).

31. H. W. Morsi and W. Reich, Astron. Astrophys. Suppl. Ser. 71, 189 (1987).

32. R. Kothes and W. Reich, Astron. Astrophys. 372, 627 (2001).

33. N. E. Kassim, Astron. J. 103, 943 (1992).

34. D. A. Green, S. P. Reynolds, K. J. Borkowski, U. Hwang, I. Harrus, and R. Petre, Mon. Not. R. Astron. Soc. 387, L54 (2008).

35. G. Zanardo, L. Staveley-Smith, L. Ball, B. M. Gaensler, et al., Sov. Astron. 710, 1515 (2010).

36. N. S. Kardashev, Astrophys. J. 6, 317 (1962).

37. M. S. E. Roberts, C. R. Tam, V. M. Kaspi, M. Lyutikov, G. Vasisht, M. Pivovaroff, E. V. Gotthelf, and N. Kawai, Astrophys. J. 588, 992 (2003).

Translated by E. Seifina 